\title{
Temporal evolution of relaxation in rubbed polystyrene thin films
}

\author{
O. C. Tsang, O. K. C. Tsui, ${ }^{*}$ and Z. Yang ${ }^{\dagger}$ \\ Department of Physics, The Hong Kong University of Science and Technology, Clearwater Bay, Kowloon, Hong Kong
}

(Received 28 August 2000; published 24 May 2001)

\begin{abstract}
Temporal relaxation of rubbed polystyrene (PS) thin films (50 nm thick) was studied by probing the decay of the optical anisotropy in the sample. Our results show that the relaxation process is characterized by two single-exponential decays plus a temperature-dependent constant. Both relaxations are orders of magnitude faster than the main chain $(\alpha)$ relaxation at temperatures well below $\left(>10^{\circ} \mathrm{C}\right) T_{g}$, with activation energies 3.0 $\mathrm{kcal} / \mathrm{mol}$ and $5.1 \mathrm{kcal} / \mathrm{mol}$, respectively, either of which are much smaller than that of the $\alpha$ relaxation $(\sim 50$ $\mathrm{kcal} / \mathrm{mol})$. The decay time constants are found to be independent of the sample molecular weights $M_{w}(=13.7$ $\mathrm{K}$ to $550 \mathrm{~K}$ Daltons, $\left.M_{w} / M_{n} \leqslant 1.1\right)$ at these temperatures. This shows the local nature of the relaxation modes of rubbed PS.
\end{abstract}

DOI: 10.1103/PhysRevE.63.061603

PACS number(s): 68.15.+e, 61.20.Lc, 68.60.-p

Rubbing with piled fabrics such as velour and velvet creates surface scratches [1] and molecule alignments $[2,3]$ in polymer thin films. After rubbing, the molecular alignment decreases at elevated temperatures as the polymer relaxes. The understanding of such relaxation dynamics and mechanism is of practical importance. For example, rubbed polyimide films are widely used in the liquid-crystal display industry. Reduction in molecular alignments of rubbed polymers due to relaxations may lead to failure of the device since its operation relies heavily on the integrity of the molecular alignment, yet little is known about the relaxation dynamics of rubbed polymer films. In addition to the recent findings on the different dynamical behaviors of polymer thin films compared to the bulk [4-8], the subject matter is very interesting fundamentally as well. Liu $e t$ al. used nearedge $\mathrm{x}$-ray absorption fine structure (NEXAFS) to study the absorption anisotropy of rubbed thin polystyrene (PS) films on Si covered with native oxide, and found that after annealing at an elevated temperature, some amount of anisotropy disappeared in the initial $30 \mathrm{~min}$ while the rest remained unaffected with subsequent annealing at the same temperature [3]. However, when the glass transition temperature $T_{g}$ of the polymer was reached, all of the remaining anisotropy disappeared over a short time. Recently Schwab et al. [8] studied the relaxation of rubbed PS films on glass substrates by measuring the optical anisotropy of the rubbed films as the sample was heated at a constant rate of $1^{\circ} \mathrm{C} / \mathrm{min}$. The authors suggested that the relaxation of the polymer (i.e., disappearance of the anisotropy) followed the structural relaxation dynamics of polymer backbones related to the glass transition, and could be described by the KohlrauschWilliams-Watts (KWW) stretched exponential function $[7,9]$ :

$$
\phi(t)=\phi(0) e^{-(t / \tau)^{\beta}}
$$

\footnotetext{
*Author to whom correspondence should be addressed. Electronic address: phtsui@ust.hk

†Electronic address: phyang@ust.hk
}

where $\beta$ is about 0.38 for PS. The authors further modeled $\tau$ by a simple Arrhenius relationship below the $T_{g}[8,10]$ :

$$
\tau=\tau\left(T_{g}\right) e^{\Delta E / R\left(1 / T-1 / T_{g}\right)} .
$$

Here, $\Delta E$ is the activation energy and $R$ the gas constant. Using the parameters found in Ref. [8] based on this model (i.e., $\quad \tau\left(T_{g}\right)=5 \mathrm{~s}, \quad \Delta E=205 \mathrm{~kJ} / \mathrm{mol}, \quad R=8.315 \mathrm{~J} / \mathrm{mol}, \quad T_{g}$ $=97^{\circ} \mathrm{C}$ ), the decay time constant $\tau$ at $20^{\circ} \mathrm{C}$ below $T_{g}$ is $\sim 227 \mathrm{~s}$. Annealing at such temperature for over $1,000 \mathrm{~s}$ should remove all the anisotropy. This is in contradiction with findings of Ref. [3], where a nonzero anisotropy was still detectable after 1,800 s of annealing, and the anisotropy changed little with a longer annealing time. For unrubbed PS films, Forrest et al. [7] studied the structural relaxation using photon correlation spectroscopy (PCS). The relaxation dynamics were found to follow the KWW function. Furthermore, the relaxation time $\tau$ was measured to increase by $>100$ times when the temperature was decreased by $6^{\circ} \mathrm{C}$ below the $T_{g}$, consistent with an extrapolation from data taken with the microbalance technique at temperatures $8-15^{\circ} \mathrm{C}$ above the $T_{g}$ using the Vogel-Tammann-Fulcher (VTF) relation [7]

$$
\langle\tau(T)\rangle=\tau_{0} \exp B /\left(T-T_{0}\right),
$$

where $\tau_{0}$ is the asymptotic relaxation time at high temperatures, $T_{0}$ is the Tammann temperature $\sim T_{g}-50 \mathrm{~K}$, and $B$ is a constant related to the fragility of the polymer. Assuming the same VTF relation for $\tau(T)$ at temperatures further below $T_{g}$, one may extrapolate that $\tau$ should be thousands of seconds or more at temperatures several tens of degrees below $T_{g}[7,8]$. If the relaxation of aligned PS films should follow that of unrubbed PS, no significant decrease in the sample's alignment would be expected at temperatures well below $T_{g}$. Contrary to this simple deduction, notable relaxation was found in aligned PS thin films even at temperatures $30^{\circ} \mathrm{C}$ or more below $T_{g}[3,8]$. Therefore, the assumption that relaxation dynamics of rubbed PS is the same as that of the unperturbed counterpart is not justifiable.

We report here on the study of temporal evolution of relaxation in rubbed PS thin films at elevated temperatures 
using the optical anisotropy detection technique. Major findings of the present paper are (1) there is significant amount of relaxation occurring in the sample, determined by the amount of decrease in the sample's optical anisotropy, even at temperatures tens of degrees below $T_{g} ;(2)$ the relaxation process is clearly non-KWW but is characterized by two single exponentials plus a temperature-dependent constant $C(T)$, with the fast decay time constant being only $256 \mathrm{~s}$ at $\sim 60{ }^{\circ} \mathrm{C}$ below $T_{g}$; (3) the decay time constants are the same within $20 \%$ for PS with molecular weights $M_{w}=13.7,44.1$, 115,221 , and $550 \mathrm{~K}$ Daltons, all with $M_{w} / M_{n} \leqslant 1.1$, at temperatures well below $T_{g}$; (4) the temperature dependence of both decay time constants can be fitted to the Arrhenius relation [Eq. (2)], with activation energies of 3.0 and $5.1 \mathrm{kcal} /$ mol for the fast and slow processes, respectively; and (5) the temperature at which $C(T)=0$ is close to $T_{g}$. All these point out that the relaxation of PS upon rubbing involves mostly local, noncooperative movements. Global relaxation is significant only at temperatures near $T_{g}$.

Monodispersed PS $\left(M_{w} / M_{n}<1.1\right)$ were purchased from Scientific Polymer Products (Ontario, NY) and were used without further purification. The thin films were prepared by spin coating solutions of the polymer in toluene (1-2 wt \%) onto acid-cleaned $\mathrm{Si}$ substrates with a native oxide layer $\left(\sim 1.2 \mathrm{~nm}\right.$ thick). They were then baked at $130^{\circ} \mathrm{C}$ for $5 \mathrm{~h}$. The resultant film thickness was determined to be 50 $\pm 2 \mathrm{~nm}$ using ellipsometry. Rubbing was done in a homemade apparatus with a rayon cloth mounted on a metal block. An electric motor pulls the block at a constant speed of $1 \mathrm{~cm} / \mathrm{s}$. All samples used in this paper had been rubbed repeatedly under the same pressure of $10 \mathrm{~g} / \mathrm{cm}^{2}$ until their optical anisotropy no longer increased with further rubbing.

Optical anisotropy of the rubbed samples was measured by reflectance difference spectroscopy $[11,12]$. Since all anisotropy spectra showed a maximum at $\sim 300 \mathrm{~nm}$ in wavelength, this wavelength was used in subsequent measurements. Samples were placed face up into a heating block stabilized at the measurement temperature to better than $\pm 0.1^{\circ} \mathrm{C}$ using a Lakeshore sensor-heater pair and a Model334 temperature controller. To measure the optical anisotropy vs time at temperature $T$, the heating block was preheated to $T$ before a freshly rubbed sample was placed inside the heating block. Then temporal measurements would commence immediately. Using a FSI Model-812 thermal imaging camera, we observed that the sample was in thermal equilibrium within seconds of contact with the heating block, and the temperature of the heating block was unaffected by the addition of the sample.

Figure 1 shows the optical anisotropy $\rho[\equiv \operatorname{Re}(\Delta r / r)]$ of two $50 \mathrm{~nm}$ thick PS films (one with $M_{w}=550 \mathrm{~K}$ and $M_{w} / M_{n} \sim 1.04$; the other with $M_{w}=13.7 \mathrm{~K}$ and $M_{w} / M_{n}$ $\sim 1.06)$ as a function of temperature. Here, $\Delta r$ is the complex reflectance difference between linear polarization directions parallel and perpendicular to the rubbing direction, and $r$ is the average reflectance. During the measurement, a constant heating power was applied to the block. The resultant heating rate was $\sim 6^{\circ} \mathrm{C} / \mathrm{min}$ at $20^{\circ} \mathrm{C}$ but slowed down to $\sim 0.6^{\circ} \mathrm{C} / \mathrm{min}$ at $100^{\circ} \mathrm{C}$ as the heating block approached steady thermal state. Similar to previous results $[3,8]$, our

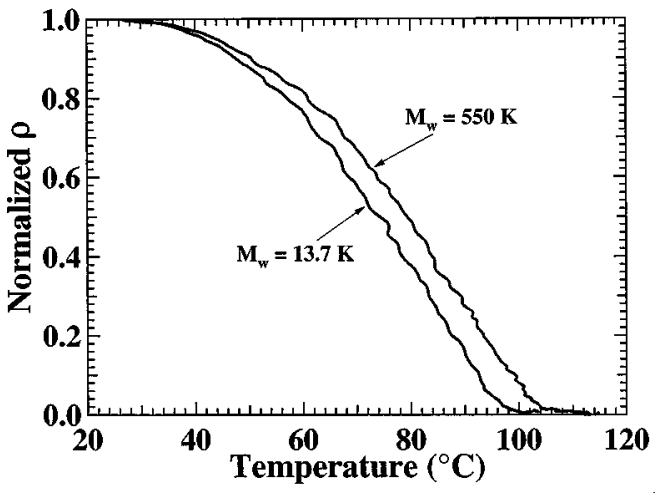

FIG. 1. The normalized anisotropy, $\rho(T) / \rho\left(T=20^{\circ} \mathrm{C}\right)$ vs temperature $T$ for a $M_{w}=550 \mathrm{~K}$ and a $M_{w}=13.7 \mathrm{KPS}$ film. Both films are $50 \mathrm{~nm}$ thick.

data show that the sample anisotropy decreases progressively as $T$ increases. At $T=40^{\circ} \mathrm{C}\left(\sim 60^{\circ} \mathrm{C}\right.$ below the $T_{g}$ of the polymer), notable relaxation is already evident, which indicates that the revealed relaxation is orders of magnitude faster than what previous measurements found [10]. On the other hand, data of Fig. 1 also show that the temperature $T_{L}$ at which the anisotropy becomes zero, occurs at $\sim 7{ }^{\circ} \mathrm{C}$ above the bulk $T_{g}$ of the polymer. In particular, for the $M_{w}$ $=550 \mathrm{~K}$ sample, $T_{L}=105^{\circ} \mathrm{C}$ and the bulk $T_{g}=98^{\circ} \mathrm{C}$ (by differential scanning calorimetry), while for the $M_{w}$ $=13.7 \mathrm{~K}$ sample, $T_{L}=98^{\circ} \mathrm{C}$ and the bulk $T_{g}=90^{\circ} \mathrm{C}$. These results suggest that the dynamical modes that have led to reduction in the sample anisotropy should, at least at temperatures near $T_{g}$, be related to the glass transition of the polymer. Ellipsometry measurements of similar polymer thin-film systems showed that the $T_{g}$ of a $50 \mathrm{~nm}$ thick PS film was comparable to that of the bulk $[4,6]$. On the other hand, it was found that the surface of a polymer could be substantially more mobile than that of the bulk [13]. If the layer of polymer modified in the rubbing process is highly localized at the free surface, the unexpectedly sizable structural relaxations observed at temperatures well below the bulk $T_{g}$ may be explained in terms of such a surface layer. The fact that $T_{L}$ corresponds well to and is slightly larger than the bulk $T_{g}$ of the polymer, however, argues against the "rubbed layer" being more mobile. This is consistent with findings in Ref. [3].

It is important to identify the origin of the optical anisotropy in order to understand what kind of relaxation our measurements indeed reveal. Atomic force microscope was employed to study the effect of rubbing on surface morphology. Freshly rubbed samples display grooves that are $\sim 50 \mathrm{~nm}$ wide and $0.8 \mathrm{~nm}$ deep. The resultant morphology-induced optical anisotropy, being proportional to the groove height, should be less than $10^{-5}$ [14], which is much less than the observed anisotropy of $5 \times 10^{-4}$. Furthermore, after the samples were heated to $90{ }^{\circ} \mathrm{C}$ for half an hour, the groove height decreased to $0.3 \mathrm{~nm}$ ( $\sim 40 \%$ drop) while the width remained unchanged. On the other hand, the measured optical anisotropy of the film decreased by more than $95 \%$ (see Fig. 1). Therefore, it is clear that the observed optical anisotropy is not due to the surface morphology but rather comes 


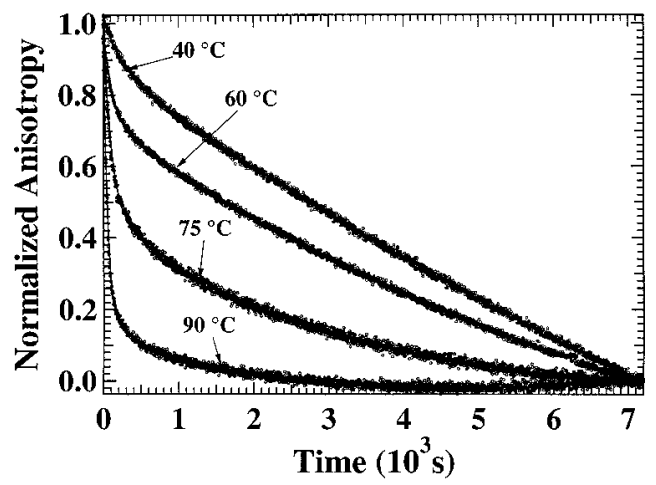

FIG. 2. Normalized background-subtracted anisotropy as a function of time at four temperatures as indicated (open circles). Solid lines are fits to the data using the double-exponential decay function [Eq. (4)].

from molecular alignments within the polymer. These observations are in consistency with previous results $[2,3,8]$. The optical anisotropy measurements should thus reveal the molecular relaxation of the polymer.

Figure 2 shows the temporal relaxation data of PS films with $M_{w}=550 \mathrm{~K}$ Daltons and thickness $=50 \mathrm{~nm}$ at four different temperatures as labeled. The vertical axis represents the background-subtracted sample anisotropy, normalized by the value at zero time. We found that the anisotropy data can be fitted well to a double-exponential function with a constant term:

$$
\rho(t)=A_{1} e^{-t / \tau_{1}}+A_{2} e^{-t / \tau_{2}}+C .
$$

An attempt to fit the data to the KWW form (Eq. 1) or to a function that contains a component with the KWW form always leads to the fitted result $\beta=1$. The fitting results are summarized in Table I. The background parameter $C$, which is not shown in the table, ranges from $30 \%$ of the total anisotropy strength at $t=0$ at $40{ }^{\circ} \mathrm{C}$ to $2 \%$ of that at $90{ }^{\circ} \mathrm{C}$. Since the anisotropy data have been normalized to their value at $t=0$, only the ratio of the decay amplitudes, $A_{2} / A_{1}$ and decay times are obtained. Evidently, the amplitude ratio $A_{2} / A_{1}$ increases whereas the time constants $\tau_{1}$ (for the slow relaxation) and $\tau_{2}$ (for the fast relaxation) decrease with increasing temperature. At temperatures below $75^{\circ} \mathrm{C}$, the slow process dominates (the amplitude of the slow one is $\sim 17$ times of the fast one at $T=40^{\circ} \mathrm{C}$ ), while at a high temperature of $90^{\circ} \mathrm{C}$, the fast process takes over. Between $T=40$ and $90{ }^{\circ} \mathrm{C}$, the time constant of the slow process $\tau_{1}$ decreases by 15 times and that of the fast process $\tau_{2}$ decreases by 5 times. Both changes are much smaller than that found previ- ously ( $>10^{4}$ times) by second-harmonic generation techniques in unrubbed PS over the same temperature range [10]. Both $\tau_{1}(T)$ and $\tau_{2}(T)$ can be fitted to the Arrhenius relation, leading to activation energies 5.1 and $3.0 \mathrm{kcal} / \mathrm{mol}$, respective. The relaxation process is unexpectedly fast even at $40{ }^{\circ} \mathrm{C}$, where the time constant of the fast relaxation mode is only $256 \mathrm{~s}$. This is in drastic contrast with the $3 \times 10^{7} \mathrm{~s}$ found at $T=47^{\circ} \mathrm{C}$ in Ref. [10], or the observation by Patterson and Carroll using PCS that very little relaxation at $60{ }^{\circ} \mathrm{C}$ in unrubbed PS was observed [15]. Single exponential fast decay (on top of a KWW one) has been reported by Lee, Jamieson and Simha [16]. However, this form of decay was only observed within $5^{\circ} \mathrm{C}$ from the $T_{g}$ of the sample. All these show that the relaxation characteristics of the rubbed PS films are very different from the unrubbed ones.

To better understand the nature of the relaxations we observed in the $M_{w}=550 \mathrm{~K}$ rubbed PS films, the decay time constants of thin films (fixed at $50 \mathrm{~nm}$ thick) with other molecular weights, namely, $M_{w}=13.7,44.1,115$, and $221 \mathrm{~K}$ Daltons, were also measured. At $T=60^{\circ} \mathrm{C}$, the same doubleexponential decay form was found for all samples of different molecular weights studied. Furthermore, both time constants were the same within $20 \%$ variation, with no systematic $M_{w}$ dependence detectable. At temperatures close to the $T_{g}$ of the $M_{w}=13.7 \mathrm{~K}$ sample, however, the decay time constant becomes obviously shorter than the other samples with higher $M_{w}$, which is attributable to the lower $T_{g}$ associated with lower $M_{w}$ (see Fig. 1). The insensitivity of the decay time constants with molecular weight at temperatures well below $T_{g}$ indicates that at these temperatures the relaxations of rubbed polymers involve only the local modes. This is consistent with the earlier suggestion that the anisotropy is due to rubbing-induced alignment in the orientation of the phenyl rings [3], so relaxation of the anisotropy can occur at temperatures much below $T_{g}$ via reorientation of the phenyl rings without involving motion of the polymer backbone. Possible mechanisms for the observed local relaxations include $\gamma$ rotational motion of the phenyl rings [17] and the thermal randomization of the phenyl ring order mediated by an intermolecular field ( $\delta$ relaxation) [17]. We note that the reported activation energy of the $\gamma$ relaxation $\sim 9$ $\mathrm{kcal} / \mathrm{mol}$ [17] is comparable to the value $3.0 \mathrm{kcal} / \mathrm{mol}$ found here for the fast mode, but that reported for the $\delta$ relaxation $\sim 1.6 \mathrm{kcal} / \mathrm{mol}$ [17] is much smaller than either activation energies of the two modes. More work is clearly needed to fully understand the origins of relaxation in rubbed PS films. At temperatures approaching $T_{g}$, the main chain $(\alpha)$ relaxation is expected to accelerate rapidly in accordance with the VTF relation [Eq. (3)], and will likely become dominating as

TABLE I. Summary of the fitting parameters for the relaxation data of $M_{w}=550 \mathrm{~K}$ polystyrene films (all $50 \mathrm{~nm}$ thick) at $T=40,60,75$, and $90^{\circ} \mathrm{C}$.

\begin{tabular}{ccccc}
\hline \hline & $40{ }^{\circ} \mathrm{C}$ & $60{ }^{\circ} \mathrm{C}$ & $75{ }^{\circ} \mathrm{C}$ & $90{ }^{\circ} \mathrm{C}$ \\
\hline$A_{2} / A_{1}$ & $0.059 \pm 0.004$ & $0.22 \pm 0.02$ & $1.04 \pm 0.04$ & $4.2 \pm 0.2$ \\
$\tau_{1}\left(10^{3} \mathrm{~s}\right)$ & $15 \pm 1$ & $7.8 \pm 0.6$ & $2.8 \pm 0.2$ & $1.1 \pm 0.1$ \\
$\tau_{2}(\mathrm{~s})$ & $256 \pm 10$ & $123 \pm 6$ & $101 \pm 6$ & $50 \pm 3$ \\
\hline \hline
\end{tabular}


the corresponding relaxation time becomes shorter than those of the local relaxation modes. At $T \sim 90{ }^{\circ} \mathrm{C}$ the relaxation time of the $\alpha$ relaxation has been found to be $\sim 4000 \mathrm{~s}$ $[7,10]$, which is undoubtedly larger than that of the two relaxation modes found at $T=90^{\circ} \mathrm{C}$ in the present experiment. This may explain why the KWW relaxation has not been observed in the present experiment, and the measured $T_{L}$ 's (Fig. 1) are slightly higher than the $T_{g}$ of the polymer.

The previous results of Refs. [3] and [8] on the relaxation of rubbed PS films versus temperature can be reconciled with the presently found double-exponential relaxation scheme. Even at low temperatures, the fast process is completed within $1000 \mathrm{~s}$ whereas $\tau_{1}$ of the slow process is $>10000 \mathrm{~s}$ (Table I). At high temperatures such as $T \sim 90{ }^{\circ} \mathrm{C}$, the fast relaxation time shortens to only $5 \mathrm{~s}$, but that of the slow relaxation time remains large $(\sim 1100 \mathrm{~s})$. That is why in the NEXAFS experiment of Ref. [3], the authors found that upon annealing the samples at a present temperature for $30 \mathrm{~min}$, the sample anisotropy was almost time independent at 10 min intervals. The relaxation vs $T$ data of Ref. [8] can also be interpreted without invoking the KWW decay process. Since the temperature was raised at a rate of $1{ }^{\circ} \mathrm{C} / \mathrm{min}$ in that experiment, the observed anisotropy vs $T$ should be fully accountable by just the fast decay process. This also explain why the $T$ dependence results we obtained (Fig. 1) at a higher heating rate (averaged $3.3^{\circ} \mathrm{C} / \mathrm{min}$ ) still looks similar to that of Ref. [8].

In summary, we have shown that the temporal evolution of relaxations in rubbed PS thin films at temperatures $40^{\circ} \mathrm{C} \leqslant T \leqslant 90^{\circ} \mathrm{C}$ did not exhibit the KWW behavior, which is typically observed near the glass-to-rubber transition temperature. Instead, the temporal relaxation fits well to a double-exponential decay function, with the activation energy of the fast and slow relaxation modes being 3.0 and 5.1 $\mathrm{kcal} / \mathrm{mol}$, respectively, much smaller than the value $50 \mathrm{kcal} /$ mol that had been found for the $\alpha$ relaxation. On the other hand, the temperature at which complete relaxation of anisotropy occurs is at $\sim 7^{\circ} \mathrm{C}$ above the $T_{g}$ of the PS, suggesting that the $\alpha$ relaxation may become dominant at temperatures near $T_{g}$. The lack of $M_{w}$ dependence of $\tau_{1}$ and $\tau_{2}$ at $T$ $=60{ }^{\circ} \mathrm{C}$ evidences the local nature of the relaxation modes at low temperatures in rubbed PS films.

We thank Dr. X. R. Wang for useful discussions, and W. T. Leung, J. Y. L. Ho, and F. K. Lee for technical assistance. O. K. C. T. acknowledges financial support by the Research Grant Council of Hong Kong through the Hong Kong University of Science and Technology under Project No. DAG98/99 S24.
[1] J-H. Kim and C. Rosenbiatt, Appl. Phys. Lett. 72, 1917 (1998).

[2] M. F. Toney, T. P. Russel, J. A. Logan, H. Kikuchi, J. M. Sands, and S. K. Kumar, Nature (London) 374, 709 (1995).

[3] Y. Liu, T. P. Russel, M. G. Samant, J. Stohr, H. R. Brown, A. Cossy-Favre, and J. Diaz, Macromolecules 30, 7768 (1997).

[4] J. L. Keddie, R. A. L. Jones, and R. A. Cory, Europhys. Lett. 27, 59 (1994).

[5] W. E. Wallace, J. H. van Zanten, and W. L. Wu, Phys. Rev. E 52, R3329 (1995).

[6] J. A. Forest, K. Dalnoki-Veress, and J. R. Dutcher, Phys. Rev. E 56, 5705 (1997).

[7] J. A. Forest, C. Svanberg, K. Revesz, M. Rodahl, L. M. Torell, and B. Kasemo, Phys. Rev. E 58, R1226 (1998).

[8] A. D. Schwab, D. M. G. Agra, J-H. Kim, S. Kumar, and A. Dhinojwala, Macromolecules 33, 4903 (2000).

[9] G. Williams and D. C. Watts, Trans. Faraday Soc. 66, 80
(1970).

[10] A. Dhinojwala, G. K. Wong, and J. M. Torkelson, J. Chem. Phys. 100, 6046 (1994).

[11] D. E. Aspnes, J. P. Harbison, A. A. Studna, and L. T. Florez, J. Vac. Sci. Technol. A 6, 1327 (1988).

[12] Z. Yang, G. K. L. Wong, I. K. Sou, and Y. H. Yeung, Appl. Phys. Lett. 66, 2235 (1995).

[13] K. Tanaka, A. Takahara, and T. Kajiyama, Macromolecules 30, 6626 (1997).

[14] D. E. Aspnes, Phys. Rev. B 41, 10334 (1990).

[15] G. D. Patterson and P. J. Carroll, J. Polym. Sci., Polym. Phys. Ed. 21, 1897 (1983).

[16] H. Lee, A. M. Jamieson, and R. Simha, Macromolecules 12, 329 (1979).

[17] O. Yano, and Y. Wada, J. Polym. Sci:Part A 9, 669 (1971). 